protacted disease course and a failure to improve. ${ }^{5}$ Acute inflammatory demyelinating neuropathy in association with acute leukaemia has been reported in only a few instances. ${ }^{1-3}$ However, in contrast to our patient, GBS in these cases was rapid progressive and the patients died in a few weeks without haematological remission. ${ }^{1-3}$ This case is remarkable because the associated neurological syndrome apparently did not adversely affect the therapeutic response and the prognosis of the myeloproliferative disorder. The reasons for this difference are not clear. The neuropathy in these cases is usually attributed to leukaemic infiltrations. ${ }^{1-3}$ However, remote effects probably related to transient immunosuppression in acute leukaemia could serve as one contributing factor in triggering Guillain-Barré syndrome in some patients. ${ }^{3}$ The findings described in our case tend to support this view.

$$
\begin{array}{r}
\text { A RODRIGUES } \\
\text { A MONTEIRO } \\
\text { J VIANA } \\
\text { A MACEDO } \\
\text { F GRACA } \\
\text { A SENA } \\
\text { Haematology Unit and Neurology Service, } \\
\text { Hospital de St António dos Capuchos, } \\
1100 \text { Lisbon, Portugal. }
\end{array}
$$

Correspondence to: Professor Sena, Neurology Service.

1 McLeod JG, Walsh JC. Peripheral neuropathy associated with lymphomas and other reticuloses. In: Dyck PJ, Thomas PK, Lambert EH, Bunge R, eds. Peripheral neuropathy, vol 2. Philadelphia: WS Saunders, 1984: 2192-203.

2 Krendel DA, Albright RE, Graham DG. Infiltrative polyneuropathy due to acute monoblastic leukemia in hematologic remission. Neurology 1987;37:474-7.

3 Phanthumchinda $K$, Intragumtornchai $T$, Kasantikul V. Guillain-Barré syndrome and optic neuropathy in acute leukemia. optic neuropathy in acut

4 Benett JM, Catovsky D, Daniel MT, Flandrin G, Galton DAG, Gralnick H, Sultan C. Gr, Galton DAG, Gralnick $H$, Sultan C. Proposed revised criteria for the classifica-
tion of acute myeloid leukemia. Ann Intern tion of acute myeloid

5 Halls J, Bredkyaer C, Friis ML. Guillain-Barré syndrome: diagnostic criteria, epidemiology, clinical course and prognosis. Acta Neurol Scand 1988;78:118-122.

Polyunsaturated fatty acids in peroxisomal disorders: a hypothesis and a proposal for treatment

Defects of peroxisomal $\beta$-oxidation of fatty acids are characteristic of a group of congenital disorders with severe disturbances of brain function, or of the spinal cord, such as the cerebro-hepato-renal syndrome of Zellweger, infantile Refsum's disease, neonatal adrenoleukodystrophy, X-linked adrenoleukodystrophy and adrenomyeloneuropathy, hyperpipecolic acidaemia, isolated absence of an enzyme of the $\beta$-oxidation sequence, and several variants described clinically as "Zellweger-like" and "neonatal adrenoleukodystrophy-like". ${ }^{1}$ In all these syndromes very long chain fatty acids (C-24, C-26) accumulate in plasma. Medical treatment at present succeeds in normalising their level, by suppressing endogenous synthesis by glycerol trioleate and glycerol trierucate administration; but it is doubtful whether this leads to clinical improvement.

It has been shown recently that brain, retina, liver, kidney and erythrocytes of patients with several peroxisomal disorders are depleted of docosahexaenoic acid C 22:6 63 (DHA), a component which normally rises in the brain and retina during prenatal and postnatal development. ${ }^{2}$ Depletion of DHA in the rhesus monkey as well as in children causes disturbances of photoreceptor function (ERG, visual acuity) and peripheral neuropathy. ${ }^{34}$ Martinez $^{5}$ orally administered $250 \mathrm{mg}$ of DHA ethylester daily for three months to a child with an NALD-like syndrome. DHA erythrocyte levels normalised but in addition there was improvement of psychomotor development and visually evoked potentials.

We propose that the relationship between peroxisomal $\beta$-oxidation and DHA levels is not fortuitous, but is explained by the results of Voss et al. ${ }^{6}$ These authors have shown that in the rat synthesis of C 22:6 133 proceeds by $\beta$-oxidation of $C 24: 6 \omega 3$. Obviously, oxidation of C 24 is impaired in patients with a peroxisomal $\beta$-oxidation defect, and this must result in shortage of DHA. The existence of the latter mechanism is confirmed by most recent, and unexpected, data from patients with schizophrenia treated with high doses of phenothiazines. ${ }^{7}$ These drugs are in vivo inhibitors of peroxisomal $\beta$-oxidation. The patients' thrombocytes became deficient in PUFA, especially arachidonic acid and DHA; while the ratio $C$ 26:0/22:0 increased.

We propose that: a) all patients with an impaired peroxisomal function should be assayed for PUFA levels in erythrocytes or platelets, and in biopsied liver. Brain and spinal cord will be still more informative, as their content is not necessarily reflected in blood cells. Data on X-ALD and AMN, which form the largest group of peroxisomal diseases, are still fragmentary. Although erythrocyte levels were normal in several patients, Martinez ${ }^{2}$ found the DHA in the brain of a single case to be below those of extreme nutritional deprivation; the brain of a patient with adrenomyeloneuropathy had normal PUFA; b) patients with a lowered DHA should be treated by DHA supplementation. Such treatments have been initiated by $\mathrm{Dr} M$ Martinez, under a European project "Pathogenesis, prevention and treatment of peroxisomal leukodystrophy", in which more than 30 centres in 13 countries take part. For a correct evaluation it is necessary that objective tests (MRI, neurophysiological and other tests) be carried out before treatment starts. The evolution of the PUFA level should be monitored.

FRANK ROELS Human Anatomy and Embryology,
University of Gent, Belgium SVEN FISCHER Department of Medicine II, Department of Medicine II,
University of Munich, Germany

\section{WERNER KISSLING} Department of Psychiatry,

Technical University of Munich, Germany

Correspondence to: Professor Roels, coordinator European Project on Peroxisomal Leukodystrophy, Godshuizenlaan 4, 9000 Gent, Belgium.

1 Roels F. Peroxisomes: a personal account. Brussels: VUB Press, 1991: ISBN 90-70289-94-6. 1-151.

2 Martinez M. Abnormal profiles of polyunsaturated fatty acids in the brain, liver, kidney and retina of patients with peroxisomal and retina of patients with peroxison
disorders. Brain Res 1992;583:171-82.

3 Connor WE, Neuringer $M$, Reisbick $S$. Essential fatty acids: The importance of $n-3$ Essential fatty acids: The importance of n-3
fatty acids in the retina and brain. Nutrition fatty acids in the retin
$R e v 1992 ; 50: 21-9$.

4 Birch DG, Birch EE, Hoffman DR, Uauy RD. Retinal development in very-low-birthweight infants fed diets differing in omega-3 fatty acids. Invest Ophthalmol and Visual Science 1992;33:2365-76.

5 Martinez M. Treatment with docosahexaenoic acid favorably modifies the fatty acid composition of erythrocytes in peroxisomal patients. In: Coates PM, Tanaka $\mathrm{K}$, eds. Nerw developments in fatty acid oxidation. New York: Wiley-Liss, 1992:389-97.

6 Voss A, Reinhart M, Sankarappa S, Sprecher H. The metabolism of 7,10,13,16,19docosapentaenoic acid to $4,7,10,13,16,19$ docosahexaenoic acid in rat liver is independent of a 4-desaturase. F Biol Chem
1991;266:19995-20000. Fischer S, Kissling W, Kuß H-J. Schizophrenic patients treated with high dose phenothiazine or thioxanthene become deficient in polyunsaturated fatty acids in their thrombocytes. Biochem Pharmacol 1992;44:317-23.

Herpes simplex encephalitis in a patient with complex partial epilepsy: confirmation by the polymerase chain reaction with necropsy studies

Human herpes simplex virus 1 (HSV) is a relatively common and eminently treatable cause of encephalitis. Despite its moderately distinctive clinical presentation, ${ }^{1}$ the clinical diagnosis of herpes simplex encephalitis (HSE) is unreliable, ${ }^{2}$ and until recently laboratory diagnosis of HSE has been unsatisfactory: isolation of the virus from CSF is rarely achieved; antigen detection has proved problematic, diagnosis by serology or immunoblotting must rely on the evolving immune response and is therefore delayed and indirect, and brain biopsy is highly invasive. The polymerase chain reaction (PCR) ${ }^{3}$ is a method for the amplification of DNA with exquisite sensitivity. In cases of suspected HSE it holds out the promise of early and accurate diagnosis.

We describe a case in which the eventual diagnosis of HSE by PCR was confirmed at necropsy. It illustrates the potential difficulty of making this diagnosis in a patient with pre-existing epilepsy and also exemplifies the occasional occurrence of a false negative result from PCR. This implies that negative results should be assessed critically and that repeated sampling may be worthwhile in difficult cases.

A 73 year old woman was admitted "drowsy but responsive" to her District General Hospital in 1991 following "multiple fits". In 1982 she had been investigated because of a five year history of episodic depresonalisation, micropsia, formed visual hallucinations and, rarely, loss of consciousness. Successive EEGs revealed only moderate bitemporal abnormalities, but a CT scan demonstrated a right petrous meningioma with associated intracerebral oedema. The possibility of surgery was considered but rejected. Her epilepsy was controlled by anticonvulsant therapy although she became prone to periods of prolonged postical confusion. On admission in 1991 she was drowsy but afebrile and there were no focal neurological signs. Her level of alertness improved over the following few days, although she remained confused, belligerent and possibly dysphasic. Five days after admission she had a flurry of complex partial seizures, became drowsy and febrile, and was treated with intravenous antibiotics. Three days later she was transferred to our care. On arrival she was cyanosed, febrile at $40^{\circ} \mathrm{C}$, and deeply unconscious. She was severely hypoxic and was ventilated. A chest radiograph suggested left basal consolidation. An EEG showed rhyth- 
mic slow wave discharges on the right at 1-2 Hz, occasionally associated with spikes, and more clearly epileptiform activity on the left. Intravenous diazepam abolished the activity on the left, but spared that on the right. The initial diagnosis was of pneumonia with complex partial status epilepticus. On the following day the EEG record was dominated by widespread, irregular, repetitive complexes with a period of approximately two seconds. Unenhanced CT scan appearances had not, however, changed since 1988. A lumbar puncture was performed as her conscious level had failed to improve. The CSF was under a pressure of $24 \mathrm{cms}$ and contained $52 \times 10^{6} / \mathrm{L}$ leucocytes (93\% lymphocytes) with normal CSF protein and CSF: blood glucose ratio. A further lumbar puncture two days later showed worsening abnormality and treatment was started with intravenous Acyclovir, rifampicin, isoniazid and pyrazinamide. Over the following nine days her reflex responses deteriorated and she died.

A polymerase chain reaction for the amplification of HSV-1 DNA was carried out post-mortem using the primer sequences and methods described by Aurelius et $a l^{3}$ with some modifications. Reaction conditions for first and second round PCR were as described previously. ${ }^{4}$ The patient's second CSF sample and the positive controls contained detectable HSV DNA after two rounds of PCR (fig). The first CSF sample, taken two days before, was negative. Neither oligoclonal bands of total IgG, nor antigen specific oligoclonal bands were detected in either CSF sample. Viral culture and viral titres were negative in the first CSF sample; in the second sample viral culture was again negative but a weak IgG response to Herpes simplex virus 1 was detected by ELISA.

Post mortem examination confirmed the presence of a right petrous meningioma. The brain was swollen and soft, with uncal and cerebellar tonsillar herniation. Temporal and insular cortex were involved in a marked meningoencephalitis, with necrotising venulitis and perivascular infiltration by lymphocytes and macrophages. Immunoperoxidase staining revealed a very striking positive reaction for herpes simplex virus antigens in neurons, macrophages, and many cells whose nature could not be identified.

This complex case emphasises the importance of prompt treatment with Acyclovir ${ }^{5}$ where there is clinical suspicion of the diagnosis of HSE. In general the diagnosis should be considered in any patient with fever and depression of consciousness: suspicion should be heightened by accompany-

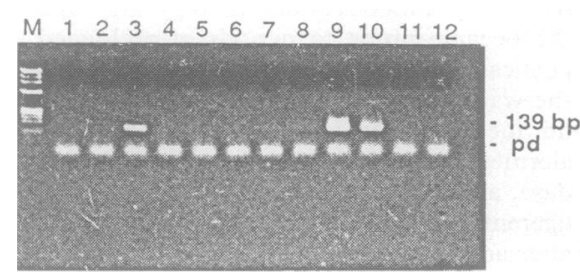

Figure Second round PCR products, on ethidium bromide stained gel, showing a positive reaction in lanes 3 (patient's CSF) and lanes 9 and 10 (positive controls). Lane $M$ contains molecular weight markers. $p d=$ primer-dimer amplification products, $139 \mathrm{bp}=$ herpes simplex virus type 1 second round PCR products. ing abnormalities of behaviour, focal seizures or signs, especially dysphasia, evidence of acute temporal lobe pathology from neuroimaging or EEG, or a CSF lymphocytosis. ${ }^{1}$ A number of factors conspired to reduce clinical suspicion in the present case, in particular the patient's long history of epilepsy with pronounced post-ictal confusion and the clinical evidence for a severe pneumonia. In retrospect, the onset of encephalopathy with drowsiness, seizures, fever and new EEG disturbance was fully in keeping with the eventual diagnosis of HSE. It is of course always important, even in the context of a patient with a chronic disorder, to assess an acute illness on its own merits.

Although PCR is highly sensitive and specific, the negative result from the first CSF sample reminds us that all laboratory tests give rise to occasional false negative results: it is advisable to test more than once in difficult cases, and a negative PCR result should not preclude the use of acyclovir where HSE is suspected clinically.

AZJ ZEMAN

K MILES

M CIARDI

Institute of Neurology, Queen Square,

JD FOX

Division of Virology, University College and Middlesex School of Medicine, London, UK

1 Whitley RJ, Soong S-J, Linneman C, Liu C, Pazin G, Alford A. Herpes simplex encephalitis: clinical assessment. $\mathscr{f} A M A$ 1982;247:317-20.

2 Whitley RJ, Cobbs CG, Alford CA, et al. Diseases that mimic herpes simplex encephalitis: diagnosis, presentation and outcome. FAMA 1989;262:234-9.

3 Aurelius E, Johansson B, Skoldenburg B, Staland A, Forsgren M. Rapid diagnosis of herpes simplex encephalitis by nested polymerase chain reaction assay of cerebrospinal merase chain reaction assay of
fluid. Lancet 1991;337:189-92.

4 Wakefield AJ, Fox JD, Sawyerr AM, et al. Detection of herpes virus DNA in the large intestine of patients with ulcerative colitis and Crohn's disease using the nested polymerase chain reaction. $\mathcal{f}$ Med Virol 1992;38:183-90.

5 Skoldenburg B, Alestig K, Burman L, et al. Acyclovir versus vidarabine in Herpes simplex encephalitis. Lancet 1984;ii:707-11.

\section{MATTERS ARISING}

\section{Accuracy of clinical diagnosis of}

idiopathic Parkinson's disease

I read the paper by Hughes et $a l^{1}$ in the journal with much interest and wish to compliment them for their work.

The percentage of inaccurate clinical diagnosis of idiopathic Parkinson's disease (IPD) in their study is identical to that which we reported last year. ${ }^{2}$ Their observations, however, are different from our study in several respects. For example, the largest subgroup of patients (6 cases) which were erroneously diagnosed as having IPD by Hughes $e t a l^{1}$ had progressive supranuclear palsy (PSP); in contrast, all the necropsy proven PSP cases in our study were recognised before death. ${ }^{2}$ The reasons for that difference are unclear. The final clinical diagnosis in all our patients was made by the same neurologist. ${ }^{2}$ Hughes et al ${ }^{1}$ did not indicate the number of neurologists and the geriatricians involved in the evaluation of their patients and those contributing to the brain bank. The larger the number of clinicians assessing the patients, the greater would be the variability and the chance of error.

The second possibility for the misdiagnosis is that the patients may have been evaluated during an early stage of illness, before the features characteristic of PSP were evident. The clinical data in our cases were collected prospectively and we were able to assess the issue of diagnostic accuracy based on each, the initial and the final clinical diagnosis before death. While only $65 \%$ of our cases whose initial diagnosis was IPD had Lewy body pathology, the diagnostic accuracy increased to $76 \%$ by the time the final assessment was done-mean 12 years after onset. Most cases who had other variants of Parkinsonian syndrome (PS) were recognised within 5 years of onset. It is unclear in the report if they ${ }^{1}$ relied on initial or the final clinical diagnosis.

Another aspect that $\mathrm{we}^{2}$ made a note of but is not clarified in their study ${ }^{1}$ is the method used to assign one diagnosis to a patient. We considered the clinical diagnosis correct only if it was the sole diagnosis or the pathological diagnosis was listed at the top of diagnostic possibilities. ${ }^{2}$

Several variants of PS, including PSP $^{3-5}$ were clinically and pathologically identified in the early 1960s. Those patients who were evaluated before that would therefore have a larger proportion of inaccurate diagnosis by contemporary standards. Their paper does not indicate if some of the errors in diagnosis may be related to the calendar year of patient assessment.

In addition to our paper, there is a small study by Forno 6 which addressed the issue of the accuracy of clinical diagnosis in IPD.

Hughes et al retrospectively analysed patients using the UK Parkinson's Disease Society Brain Bank (PDSBB) clinical diagnostic criteria. By those criteria, 11 patients did not have IPD, yet $3(27 \%)$ of those turned out to have Lewy body pathology. They have not discussed the reasons for this significant error. Whatever the reason, it illustrates that no diagnostic criteria are fool-proof.

I suggest a minor amendment to the PDSBB clinical criteria for consideration by the committee. Postural instability which has been recommended as one of the two major manifestations necessary for making the diagnosis of PS should be deleted as it is present in a large segment of normal elderly people. Most clinicians looking after elderly people recognise that individuals in this age group lose balance rather easily compared with younger age groups. Postural instability, as evaluated in the PS, was carefully studied by Weiner $e t a l$ in the elderly. When all the neurological, mechanical and other possible causes for postural reflex impairment were excluded, they noted that postural instability was an age-related phenomenon. ${ }^{7}$ While $43 \%$ of those between age 60-69 years had impaired postural reflexes, $70 \%$ between $80-89$ and $100 \%$ of those between age 90-99 years had impaired postural reflexes.?

DR RAJPUT

University of Saskatchewan,
Saskatoon, Sask, Canada

1 Hughes AJ, Daniel SE, Kilford L, Lees AJ. Accuracy of clinical diagnosis of idiopathic Parkinson's disease: A clinico-pathological study of 100 cases. $f$ Neurol Neurosurg study of 100 cases. $\mathcal{f}$
Psychiatry $1992 ; 55: 181-4$.

2 Rajput AH, Rozdilsky B, Rajput Alex H Accuracy of Clinical Diagnosis in 